Article

\title{
Unfavorable Dietary Quality Contributes to Elevated Risk of Ischemic Stroke among Residents in Southwest China: Based on the Chinese Diet Balance Index 2016 (DBI-16)
}

\author{
Yingying Wang ${ }^{1,+} \mathbb{D}, \mathrm{Xu} \mathrm{Su}^{2,+}$, Yun Chen ${ }^{1} \mathbb{D}$, Yiying Wang ${ }^{2}$, Jie Zhou ${ }^{2}$, Tao Liu ${ }^{2, *}$, Na Wang ${ }^{1, *(\mathbb{D})}$ \\ and Chaowei Fu ${ }^{1}$ (D)
}

\section{check for}

updates

Citation: Wang, Y.; Su, X.; Chen, Y.; Wang, Y.; Zhou, J.; Liu, T.; Wang, N. Fu, C. Unfavorable Dietary Quality Contributes to Elevated Risk of Ischemic Stroke among Residents in Southwest China: Based on the Chinese Diet Balance Index 2016 (DBI-16). Nutrients 2022, 14, 694. https://doi.org/10.3390/ nu14030694

Academic Editor: Panagiota Mitrou

Received: 31 December 2021

Accepted: 3 February 2022

Published: 7 February 2022

Publisher's Note: MDPI stays neutral with regard to jurisdictional claims in published maps and institutional affiliations.

Copyright: (C) 2022 by the authors. Licensee MDPI, Basel, Switzerland. This article is an open access article distributed under the terms and conditions of the Creative Commons Attribution (CC BY) license (https:// creativecommons.org/licenses/by/ $4.0 /)$.
1 Key Laboratory of Public Health Safety, NHC Key Laboratory of Health Technology Assessment, School of Public Health, Fudan University, Shanghai 200032, China; 17211020095@fudan.edu.cn (Y.W.); 18211020001@fudan.edu.cn (Y.C.); fcw@fudan.edu.cn (C.F.)

2 Guizhou Province Center for Disease Prevention and Control, Guiyang 550004, China; susuxuxu@163.com (X.S.); wyy123789123789@163.com (Y.W.); zhoujie19872014@163.com (J.Z.)

* Correspondence: liutaombs@163.com (T.L.); na.wang@fudan.edu.cn (N.W.)

+ These authors contributed equally to this work.

\begin{abstract}
Background: Little is known about the effects of dietary quality on the risk of ischemic stroke among Southwest Chinese, and evidence from prospective studies is needed. We aimed to evaluate the associations of ischemic stroke with dietary quality assessed by the Chinese Diet Balance Index 2016 (DBI-2016). Methods: The Guizhou Population Health Cohort Study (GPHCS) recruited 9280 residents aged 18 to 95 years from 12 areas in Guizhou Province, Southwest China. Baseline investigations, including information collections of diet and demographic characteristics, and anthropometric measurements were performed from 2010 to 2012. Dietary quality was assessed by using DBI-2016. The primary outcome was incident ischemic stroke diagnosed according to the International Classification of Diseases 10th revision (ICD-10) until December 2020. Data analyzed in the current study was from 7841 participants with complete information of diet assessments and ischemic stroke certification. Cox proportional hazards models were used to estimate the risk of ischemic stroke associated with dietary quality. Results: During a median follow-up of 6.63 years (range 1.11 to 9.53 years), 142 participants were diagnosed with ischemic stroke. Participants with ischemic stroke had a more excessive intake of cooking oils, alcoholic beverages, and salt, and had more inadequacy in meats than those without ischemic stroke. $(p<0.05)$. Compared with participants in the lowest quartile (Q1), those in the highest quartile (Q4) of the higher bound score (HBS) and of the dietary quality distance (DQD) had an elevated risk for ischemic stroke, with the corresponding hazard ratios (HRs) of 3.31 (95\%CI: 1.57-6.97) and 2.26 (95\%CI: 1.28-4.00), respectively, after adjustment for age, ethnic group, education level, marriage status, smoking and waist circumference, and the medical history of diabetes and hypertension at baseline. In addition, excessive intake levels (score 1-6) of cooking oils, excessive intake levels (score 1-6) of salt, and inadequate intake levels (score -12 to -7 ) of dietary variety were positively associated with an increased risk for ischemic stroke, with the multiple HRs of 3.00 (95\%CI: 1.77-5.07), 2.03 (95\%CI: 1.33-3.10) and 5.40 (95\%CI: 1.70-17.20), respectively. Conclusions: Our results suggest that unfavorable dietary quality, including overall excessive consumption, excessive intake of cooking oils and salt, or under adequate dietary diversity, may increase the risk for ischemic stroke.
\end{abstract}

Keywords: dietary quality; ischemic stroke; Chinese Diet Balance Index 2016; DBI-16; Southeast China

\section{Introduction}

Stroke is the second leading cause of death and disability worldwide, and has been the first leading cause of death and the leading cause of all-age disability-adjusted life years 
(DALYs) in China [1,2]. Notably, nationwide studies and periodic governmental reports revealed a great burden of stroke in China, with an increasing prevalence and incidence in the past decade [3,4]. According to the latest annual report in 2019, China currently has 21 million patients with stroke [5]. Ischemic stroke, defined as the permanent infarction of cerebral tissues due to abrupt decreases in cerebral blood flow, accounts for more than $70 \%$ of prevalences among all sub-types of stroke. A large prospective cohort study based on half a million Chinese adults showed that the incidence of ischemic stroke during 7.2-year follow-up was 5.86 per 1000 person-years [6].

Dietary intake is a modifiable lifestyle behavior closely associated with most noncommunicable diseases (NCDs), including cardiovascular diseases (CVDs) [7-9]. There has been an increasing interest in using specific indexes to evaluate dietary quality and their effects on NCDs, especially in some developed countries, such as the Healthy Eating Index (HEI) and the Diet Quality Index (DQI) developed for Americans [10,11], and the Mediterranean Diet Score (MDS) used for the residents in Northern Europe [12]. With reference to the methods of HEI and DQI, the Chinese Dietary Balance Index (DBI) has been designed to assess the overall diet quality in the Chinese population [13], according to the most recent Dietary Guidelines for Chinese residents [14]. Although associations of DBI with diabetes, hypertension, and cardiometabolic risk factors have been reported in previous cross-sectional studies among subgroups in China [15-17], data on the relationship between DBI and ischemic stroke is inconclusive, and evidence from prospective studies are warranted.

In this current study, we aimed to explore the associations between diet quality assessed by DBI and risk of incident ischemic stroke based on a prospective cohort in Guizhou Province in Southwest China, a region where economy and culture are relatively underdeveloped and with a great disease burden of ischemic stroke [18], in order to provide some evidence on further dietary intervention to manage and prevent ischemic stroke.

\section{Methods}

\subsection{Study Design and Participants}

The Guizhou Population Health Cohort Study (GPHCS) is one of few large populationbased prospective cohort studies in Southwest China, which was established from 12 areas (five urban districts and seven rural counties) in Guizhou Province between 20 November 2010 and 19 December 2012. A multistage proportional stratified cluster sampling method was used to obtain a representative sample of the general population in Guizhou Province. The inclusive criteria were as follows: (1) age of 18 years or above; (2) living in the study regions for more than six months and having no plan to move out; (3) completing survey questionnaire and blood sampling; (4) signing the written informed consent. A total of 9280 local residents were enrolled in the cohort. All participants were followed up for major chronic diseases and vital status through a repeated investigation conducted between 2016 and 2020. All deaths were confirmed by the record from Death Registration Information System and Basic Public Health Service System. Ethics approval was obtained from the ethics review board of Guizhou Province (No.S2017-02). All participants provided written informed consent at enrollment.

In this study, we excluded participants with a history of ischemic stroke, haemorrhagic stroke, myocardial infarction or other cardiovascular diseases, missing data of diet consumption or cardiovascular diseases at baseline, loss to follow-up, and death, leaving 7841 participants for the analyses (Figure 1).

\subsection{Outcome Definition}

The primary outcome was the first onset of ischemic stroke (I63) diagnosed according to the International Classification of Diseases 10th revision (ICD-10). All reported events were reviewed and integrated centrally by trained clinical staff. Each participant was followed up until the first occurrence of the corresponding outcome, death, or loss to 
follow-up, which occurred first before 31 December 2020. The incidence rate was calculated as the number of incident cases divided by follow-up person-years.

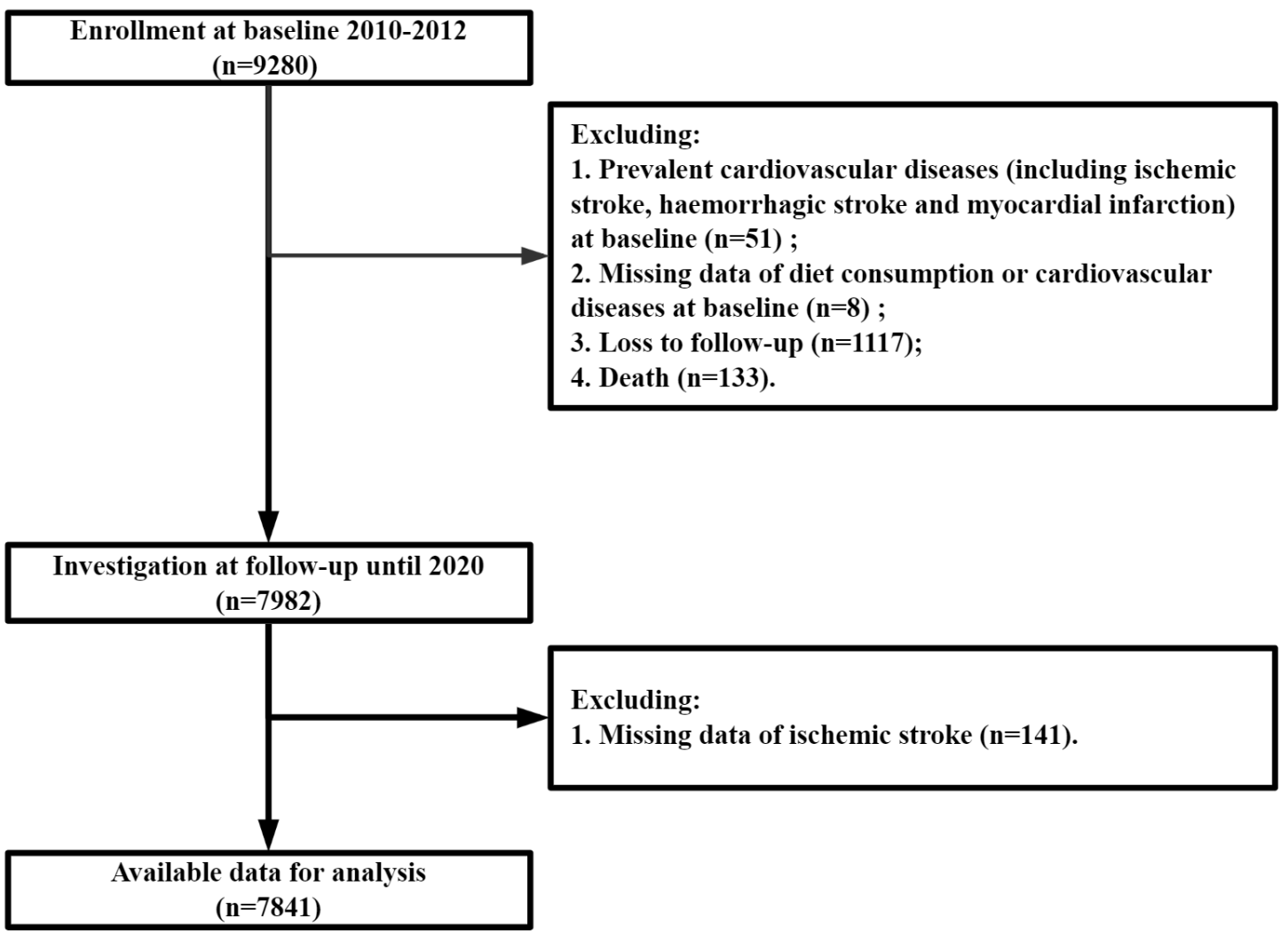

Figure 1. Flow chart of the study.

\subsection{Dietary Data Collection}

The information of dietary intake for each participant was assessed using a semiquantitative food frequency questionnaire (FFQ), both at the individual level and at the household level. The individual FFQ covered 23 items of foods and beverages which were commonly consumed, including cereals, tubers, livestock meats, poultry meats, aquatic products, vegetables, fruits, eggs, dairy products, soybean products, etc. A commonly used unit or portion size was specified for each food item, participants were required to answer their usual consuming frequency (daily, weekly, monthly, yearly, or never) of each specific food or beverages over the past one year and the amount of consumption at each time. The daily intake on average for each food item was then calculated according to the product of the intake frequency and the amount consumed at each time (in gram per day, g/day). House condiment consumption, such as cooking oils, salt, sugar, sauces, etc., was determined by evaluating all condiments consumed by all household members for one month. The total amount of condiments consumed in the household divided by the number of members usually eating at home was used to assess individual consumption of condiments.

\subsection{Dietary Intake Assessment}

The dietary quality among the participants at baseline was assessed by the Chinese Diet Balance Index 2016 (DBI-16) [13], a revised version from the Chinese Diet Balance Index 2007 (DBI-07) [19]. DBI-2016 comprises 14 subgroups of 8 components from the Dietary Guidelines for Chinese residents [14], including: (1) cereal; (2) vegetable and fruit; (3) dairy and soybean; (4) animal food (red meats/products/poultry/game, fish/shrimp, and egg); (5) empty energy foods (cooking oils, and alcoholic beverage); (6) condiments (addible sugar, and salt); (7) diet variety; and (8) drinking water. A score of 0 for each DBI-16 
component means that the individual has reached the recommended intake amounts of the corresponding food group. Positive scores (ranging 1 to 12) indicate the excessive intake level of cereals, red meat/products/poultry/game, eggs, cooking oils, alcoholic beverage, addible sugar, salt, while negative scores (ranging -12 to -1 ) indicate the inadequate intake level of cereals, vegetables, fruits, dairy, soybeans, red meat/products/poultry/game, fish/shrimps, eggs, diet variety, and drinking water. Considering the difference of nutrient requirements in energy consumption, the scoring of these 14 food subgroups was based on 11 levels of energy intake. Scoring details of DBI-16 are shown in Table S1.

Based on the scores for each DBI-16 component, three indicators of diet quality were calculated: (1) the lower bound score (LBS), an indicator for inadequate food intake, was computed by adding all the negative scores; (2) the higher bound score (HBS), an indicator for excessive food intake, was calculated by adding all the positive scores; (3) the diet quality distance, an indicator of unbalanced food intake, is calculated by adding the absolute values of both positive and negative scores [13]. The ranges of LBS, HBS, and DQD were 0 to 60, 0 to 40 , and 0 to 84 , respectively. For simplicity, each indicator was further divided into five levels to reflect the diet quality: (1) no problem, a score of 0 ; (2) almost no problem, less than $20 \%$ of the total score; (3) low-level problem, between $20 \%$ and $40 \%$ of the total score; (4) moderate level problem, between $40 \%$ and $60 \%$ of the total score; and (5) high-level problem, greater than $60 \%$ of the total score.

\subsection{Other Variables Collection}

A standardized questionnaire was used to collect the information of demographic characteristics, lifestyles, and medical history, including age, sex, area, ethnic group, education level, family income, marriage status, occupation status, physical activity, smoking or not, alcohol drinking or not, medical history of diabetes, hypertension and dyslipidemia, use of medications and nutraceuticals. Smoking was defined as smoking at least one cigarette a day for 12 months or more. Alcohol drinking was defined as drinking at least three times a week for 12 months or more. Medication use was defined as taking medications for diabetes, hypertension, dyslipidemia, or obesity regularly. Nutraceutical intake was defined as intaking some common nutraceuticals (such as vitamins or minerals), or foods with health-care functions (such as wine, tea) at least one time a week for 12 months or more. The physical activity level was calculated as the product of the duration and frequency of each activity, weighted by an estimate of the metabolic equivalent (MET) of that activity and summed for all activities performed, with the result expressed as the average MET hours per day.

Height, weight, and waist circumference were determined by trained technicians, using calibrated instruments with standard protocols and recorded to the nearest $0.1 \mathrm{~cm}$ or $0.1 \mathrm{~kg}$. Waist-to-height ratio (WHtR) was calculated as waist circumference in centimeters divided by height in meters. Body mass index (BMI) was calculated as weight in kilograms divided by the square of height in meters. Systolic blood pressure (SBP) and diastolic blood pressure (DBP) were measured from the left arm after the participant rested in a seated position. All participants provided a $10-\mathrm{mL}$ blood sample after an overnight fast of at least $10 \mathrm{~h}$, they were also required to undergo an oral glucose tolerance test (OGTT), and the plasma was obtained at $2 \mathrm{~h}$ during the test. Fasting plasma glucose (FPG), 2-h postload glucose (2h-PG) and Hemoglobin A1c (HbA1c) were determined by the glucose oxidase methods (Roche Diagnostics, Mannheim, Germany). Serum triglycerides (TG), total cholesterol (CHOL), low-density lipoprotein cholesterol (LDL-C), and high-density lipoprotein cholesterol (HDL-C) were measured by enzymatic methods (Roche Diagnostics, Mannheim, Germany).

Diabetes was defined as those above the threshold of glycemia (FPG $\geq 6.1 \mathrm{mmol} / \mathrm{L}$ or $2 \mathrm{~h}-\mathrm{PG} \geq 7.8 \mathrm{mmol} / \mathrm{L}$ ), having a reported diabetes history, or experiencing anti-diabetes medications [20]. Hypertension was defined as an abnormal level of current blood pressure (SBP > $140 \mathrm{mmHg}$ or DBP > $90 \mathrm{mmHg}$ ), having a reported hypertension history, or experiencing anti-hypertension medications [20]. Dyslipidemia was defined as an abnormal level 
of current blood lipids (TG $\geq 1.7 \mathrm{mmol} / \mathrm{L}, \mathrm{CHOL} \geq 5.2 \mathrm{mmol} / \mathrm{L}, \mathrm{LDL} \geq 3.4 \mathrm{mmol} / \mathrm{L}, \mathrm{HDL}$ $<1.0 \mathrm{mmol} / \mathrm{L}$ ), having a reported dyslipidemia history, or experiencing anti-dyslipidemia medications [20]. General overweight or obesity was defined as BMI $\geq 24 \mathrm{~kg} / \mathrm{m}^{2}$, central obesity was defined as WC $\geq 85 \mathrm{~cm}$ for females or $\geq 90 \mathrm{~cm}$ for males, and obesity status was defined as having either of these two types of obesity [21].

\subsection{Statistical Analysis}

Continuous variables were expressed as means and standard deviations (mean $\pm \mathrm{SD}$ ) and compared by using the Student's $t$-test. Categorical variables were presented as frequencies and percentages $(n, \%)$ and compared by using the Chi-square test. Considering that the proportional hazards assumption showed no strong evidence of departure, cox proportional hazards models were used to estimate hazard ratios (HRs) and 95\% confidence intervals (95\%CIs) for ischemic stroke by the components of DBI-16 and the indicators of diet quality. The level of statistical significance was defined as $\alpha=0.05$ of two-side probability. All analyses were performed using the R program (version 4.0.4, R Foundation for Statistical Computing, Vienna, Austria), and all figures were performed by using GraphPad Prism software (version 9, GraphPad Prism, San Diego, CA, USA).

\section{Results}

\subsection{Descriptions of Study Population}

The baseline characteristics of all 7841 participants in this study are shown in Table 1. The mean age was $44.18 \pm 14.97$ years at enrollment, and $47.4 \%(n=3719)$ were male. Of these, $67.1 \%(n=5258)$ of participants were rural residents, and more than half were Han Chinese $(58.5 \%, n=4589)$ and farmers $(57.3 \%, n=4490)$. The majority had an education level below junior middle school or no formal educated $(86.7 \%, n=6799)$.

Table 1. Baseline Characteristics of participants according to ischemic stroke status.

\begin{tabular}{|c|c|c|c|c|}
\hline Characteristics & $\begin{array}{c}\text { All } \\
(n=7841)\end{array}$ & $\begin{array}{c}\text { Non-Ischemic } \\
\text { Stroke } \\
(n=7699)\end{array}$ & $\begin{array}{l}\text { Ischemic Stroke } \\
\qquad(n=142)\end{array}$ & $p$ Value \\
\hline Age $(n, \%)$ & & & & $<0.001$ \\
\hline $18-40$ years & $3252(41.5)$ & $3231(42.0)$ & $21(14.8)$ & \\
\hline $41-60$ years & $3322(42.4)$ & $3256(42.3)$ & $66(46.5)$ & \\
\hline$\geq 60$ years & $1267(16.1)$ & $1212(15.7)$ & $55(38.7)$ & \\
\hline $\operatorname{Sex}(n, \%)$ & & & & 0.754 \\
\hline Male & $3719(47.4)$ & $3654(47.5)$ & $65(45.8)$ & \\
\hline Female & $4122(52.6)$ & $4045(52.5)$ & $77(54.2)$ & \\
\hline Area $(n, \%)$ & & & & 0.136 \\
\hline Urban & $2583(32.9)$ & $2545(33.1)$ & $38(26.8)$ & \\
\hline Rural & $5258(67.1)$ & $5154(66.9)$ & $104(73.2)$ & \\
\hline Ethnic group $(n, \%)$ & & & & 0.003 \\
\hline Ethnic Han & $4589(58.5)$ & $4488(58.3)$ & $101(71.1)$ & \\
\hline Minority & $3252(41.5)$ & $3211(41.7)$ & $41(28.9)$ & \\
\hline Education $(n, \%)$ & & & & 0.038 \\
\hline No formal education & $1606(20.5)$ & $1565(20.3)$ & $41(28.9)$ & \\
\hline Junior middle school and below & $5193(66.2)$ & $5111(66.4)$ & $82(57.7)$ & \\
\hline Senior high school and above & $1042(13.3)$ & $1023(13.3)$ & $19(13.4)$ & \\
\hline Family income $(n, \%)$ & & & & 0.010 \\
\hline$<3000 \mathrm{RMB} /$ person & $1664(32.6)$ & $1625(32.6)$ & $39(33.9)$ & \\
\hline 3000-10,000 RMB/person & $2129(41.8)$ & $2080(41.7)$ & $49(42.6)$ & \\
\hline$\geq 10,000 \mathrm{RMB} /$ person & $1306(25.6)$ & $1279(25.7)$ & $27(23.5)$ & \\
\hline Marriage $(n, \%)$ & & & & 0.001 \\
\hline Married/Cohabit & $6340(80.9)$ & $6226(80.9)$ & $114(80.3)$ & \\
\hline Unmarried/Single & $744(9.5)$ & $740(9.6)$ & $4(2.8)$ & \\
\hline Divorced/Widowed/Separated & $757(9.7)$ & $733(9.5)$ & $24(16.9)$ & \\
\hline
\end{tabular}


Table 1. Cont.

\begin{tabular}{|c|c|c|c|c|}
\hline Characteristics & $\begin{array}{c}\text { All } \\
(n=7841)\end{array}$ & $\begin{array}{c}\text { Non-Ischemic } \\
\text { Stroke } \\
(n=7699)\end{array}$ & $\begin{array}{l}\text { Ischemic Stroke } \\
\qquad(n=142)\end{array}$ & $p$ Value \\
\hline Occupation $(n, \%)$ & & & & 0.284 \\
\hline Farmers & $4490(57.3)$ & $4407(57.2)$ & $83(58.5)$ & \\
\hline Others & $2092(26.7)$ & $2061(26.8)$ & $31(21.8)$ & \\
\hline Unemployed or retired & $1259(16.1)$ & $1231(16.0)$ & $28(19.7)$ & \\
\hline Smoking $(n, \%)$ & & & & 0.375 \\
\hline No & $5856(74.7)$ & $5755(74.7)$ & $101(71.1)$ & \\
\hline Yes & $1985(25.3)$ & $1944(25.3)$ & $41(28.9)$ & \\
\hline Alcohol drinking $(n, \%)$ & & & & 0.403 \\
\hline No & $6038(77.0)$ & $5924(76.9)$ & $114(80.3)$ & \\
\hline Yes & $1803(23.0)$ & $1775(23.1)$ & $28(19.7)$ & \\
\hline Diabetes $(n, \%)$ & & & & 0.015 \\
\hline No & $7162(91.7)$ & $7042(91.8)$ & $120(85.7)$ & \\
\hline Yes & $648(8.3)$ & $628(8.2)$ & $20(14.3)$ & \\
\hline Hypertension $(n, \%)$ & & & & $<0.001$ \\
\hline No & $5835(74.4)$ & $5756(74.8)$ & $79(55.6)$ & \\
\hline Yes & $2006(25.6)$ & $1943(25.2)$ & $63(44.4)$ & \\
\hline Dyslipidemia $(n, \%)$ & & & & 0.581 \\
\hline No & $3353(42.8)$ & $3296(42.8)$ & $57(40.1)$ & \\
\hline Yes & $4488(57.2)$ & $4403(57.2)$ & $85(59.9)$ & \\
\hline Obesity $(n, \%)$ & & & & 0.376 \\
\hline No & $4794(64.1)$ & $4716(64.1)$ & $78(60.0)$ & \\
\hline Yes & $2688(35.9)$ & $2636(35.9)$ & $52(40.0)$ & \\
\hline Medication use $(n, \%)$ & & & & $<0.001$ \\
\hline No & $6919(88.2)$ & $6812(88.5)$ & $107(75.4)$ & \\
\hline Yes & $922(11.8)$ & $887(11.5)$ & $35(24.6)$ & \\
\hline Nutraceutical intake $(n, \%)$ & & & & 0.044 \\
\hline No & $6944(88.7)$ & $6810(88.6)$ & $134(94.4)$ & \\
\hline Yes & $883(11.3)$ & $875(11.4)$ & $8(5.6)$ & \\
\hline MET (per day, mean \pm SD) & $109.82 \pm 122.62$ & $109.87 \pm 122.68$ & $107.17 \pm 120.00$ & 0.795 \\
\hline $\mathrm{WC}(\mathrm{cm}$, mean $\pm \mathrm{SD})$ & $7661 \pm 9.46$ & $76.57 \pm 9.46$ & $78.67 \pm 9.63$ & 0.013 \\
\hline WHtR & $5.52 \pm 10.15$ & $5.50 \pm 10.15$ & $6.83 \pm 9.76$ & 0.182 \\
\hline $\mathrm{BMI}\left(\mathrm{kg} / \mathrm{m}^{2}\right.$, mean $\left.\pm \mathrm{SD}\right)$ & $22.90 \pm 3.36$ & $22.89 \pm 3.36$ & $23.26 \pm 3.25$ & 0.203 \\
\hline $\mathrm{FPG}(\mathrm{mmol} / \mathrm{L}$, mean $\pm \mathrm{SD})$ & $5.25 \pm 1.26$ & $5.25 \pm 1.25$ & $5.40 \pm 1.51$ & 0.158 \\
\hline $2 \mathrm{~h}-\mathrm{PG}(\mathrm{mmol} / \mathrm{L}$, mean $\pm \mathrm{SD})$ & $5.79 \pm 2.25$ & $5.79 \pm 2.25$ & $6.12 \pm 2.52$ & 0.088 \\
\hline $\mathrm{SBP}(\mathrm{mmHg}$, mean $\pm \mathrm{SD})$ & $125.09 \pm 20.87$ & $124.90 \pm 20.72$ & $135.33 \pm 25.97$ & $<0.001$ \\
\hline $\mathrm{DBP}(\mathrm{mmHg}$, mean $\pm \mathrm{SD})$ & $78.24 \pm 11.90$ & $78.16 \pm 11.85$ & $82.56 \pm 13.88$ & $<0.001$ \\
\hline $\mathrm{TG}(\mathrm{mmol} / \mathrm{L}$, mean $\pm \mathrm{SD})$ & $1.76 \pm 1.57$ & $1.75 \pm 1.56$ & $1.89 \pm 1.92$ & 0.324 \\
\hline $\mathrm{CHOL}(\mathrm{mmol} / \mathrm{L}$, mean $\pm \mathrm{SD})$ & $4.79 \pm 1.32$ & $4.79 \pm 1.31$ & $4.85 \pm 1.55$ & 0.64 \\
\hline $\mathrm{HDL}-\mathrm{C}(\mathrm{mmol} / \mathrm{L}$, mean $\pm \mathrm{SD})$ & $1.45 \pm 0.56$ & $1.45 \pm 0.56$ & $1.41 \pm 0.63$ & 0.405 \\
\hline $\mathrm{LDL}-\mathrm{C}(\mathrm{mmol} / \mathrm{L}$, mean $\pm \mathrm{SD})$ & $2.66 \pm 1.18$ & $2.66 \pm 1.18$ & $2.54 \pm 1.30$ & 0.239 \\
\hline
\end{tabular}

Abbreviation: SD, standard deviation; MET, metabolic equivalent of task; WC, waist circumference; WHtR, waistto-height ratio; BMI, body mass index; FPG, fasting plasma glucose; $2 \mathrm{~h}-\mathrm{PG}$, 2-h postload glucose; SBP, systolic blood pressure; DBP, diastolic blood pressure, TG, triglyceride; $\mathrm{CHOL}$, total cholesterol; HDL-C, high-density lipoprotein cholesterol; LDL-C, low-density lipoprotein cholesterol.

During a median follow-up of 6.63 years (range 1.11 to 9.53 years), 142 participants were diagnosed with ischemic stroke. Compare with participants without ischemic stroke, those with incident ischemic stroke were more likely to be older, Han Chinese, with lower economic level, and less likely to be formally educated, and married (divorced/widowed/separated). Those with ischemic stroke also tended to have prevalent diabetes and hypertension $(p<0.001)$.

\subsection{Assessments of Dietary Quality}

The distributions of scores for the DBI-16 components are presented in Table 2. Overall, $0.3 \%$ to $98.9 \%$ of participants have reached the recommended dietary intakes $($ score $=0$ ) of 
the DBI-16 components, and the majority (over 90\%) consumed appropriate amounts of addible sugar and alcoholic beverages. Inadequate intakes (score $<0$ ) were most commonly observed in dairy, fish, fruits, eggs, vegetables and soybeans, with the corresponding proportions among all participants of $99.7 \%, 97.5 \%, 95.5 \%, 83.5 \%, 62.0 \%$, and $54.4 \%$, respectively. Over $84.8 \%$ of individuals had a dietary variety below the recommended level. By contrast, excessive intakes (score $>0$ ) in cereals, cooking oils, salt and meats were also observed among $72.5 \%, 64.1 \%, 60.8 \%$, and $46.6 \%$ of participants, respectively. Participants with ischemic stroke had more excessive intake in cooking oils, alcoholic beverages, and salt, and were more likely to have inadequate intake in meats than those without ischemic stroke. $(p<0.05)$.

Table 2. Distributions of scores for the DBI-16 components and the percentages of participants with each score.

\begin{tabular}{|c|c|c|c|c|c|c|c|c|c|c|c|c|c|c|c|c|}
\hline \multirow[b]{2}{*}{ Components } & \multirow[b]{2}{*}{ Score Range ${ }^{\text {a }}$} & \multirow[b]{2}{*}{ Group } & \multicolumn{13}{|c|}{ Distribution of Score (\%) } & \multirow[b]{2}{*}{$p$ Value $^{\mathrm{b}}$} \\
\hline & & & $\begin{array}{l}(-12) \\
(-11)\end{array}$ & $\begin{array}{c}-(-10) \\
(-9)\end{array}$ & $\begin{array}{c}-(-8)- \\
(-7)\end{array}$ & $\begin{array}{l}(-6)- \\
(-5)\end{array}$ & $\begin{array}{l}(-4)- \\
(-3)\end{array}$ & $\begin{array}{l}(-2)- \\
(-1)\end{array}$ & 0 & $\begin{array}{l}(1)- \\
(2)\end{array}$ & $\begin{array}{l}\text { (3)- } \\
\text { (4) }\end{array}$ & $\begin{array}{l}\text { (5)- } \\
(6)\end{array}$ & $\begin{array}{l}\text { (7)- } \\
(8)\end{array}$ & $\begin{array}{l}\text { (9)- } \\
(10)\end{array}$ & $\begin{array}{l}\text { (11)- } \\
\text { (12) }\end{array}$ & \\
\hline \multirow[t]{2}{*}{ Cereals } & \multirow[t]{2}{*}{$(-12)-(12)$} & $\begin{array}{l}\text { Non-Ischemic } \\
\text { stroke }\end{array}$ & 0.6 & 0.8 & 1.4 & 2.2 & 4.8 & 1.7 & 16.0 & 1.1 & 18.2 & 8.8 & 5.6 & 2.9 & 36.0 & \multirow[t]{2}{*}{0.296} \\
\hline & & Ischemic stroke & 0 & 1.4 & 0.7 & 0.7 & 5.6 & 1.4 & 16.2 & 2.8 & 13.4 & 5.6 & 7.7 & 2.1 & 42.3 & \\
\hline \multirow[t]{2}{*}{ Vegetables } & \multirow[t]{2}{*}{$(-6)-(0)$} & $\begin{array}{l}\text { Non-Ischemic } \\
\text { stroke }\end{array}$ & & & & 4.2 & 26.3 & 31.5 & 38.0 & & & & & & & \multirow[t]{2}{*}{0.249} \\
\hline & & Ischemic stroke & & & & 1.4 & 23.2 & 35.9 & 39.4 & & & & & & & \\
\hline \multirow{2}{*}{ Fruits } & \multirow{2}{*}{$(-6)-(0)$} & $\begin{array}{l}\text { Non-Ischemic } \\
\text { stroke }\end{array}$ & & & & 49.0 & 39.4 & 7.1 & 4.5 & & & & & & & \multirow{2}{*}{0.325} \\
\hline & & Ischemic stroke & & & & 46.5 & 45.8 & 4.9 & 2.8 & & & & & & & \\
\hline \multirow[t]{2}{*}{ Dairy } & \multirow[t]{2}{*}{$(-6)-(0)$} & $\begin{array}{l}\text { Non-Ischemic } \\
\text { stroke }\end{array}$ & & & & 91.3 & 6.2 & 2.3 & 0.3 & & & & & & & \multirow[t]{2}{*}{0.167} \\
\hline & & Ischemic stroke & & & & 88.7 & 5.6 & 4.9 & 0.7 & & & & & & & \\
\hline \multirow[t]{2}{*}{ Soybeans } & \multirow{2}{*}{$(-6)-(0)$} & $\begin{array}{l}\text { Non-Ischemic } \\
\text { stroke }\end{array}$ & & & & 31.0 & 12.8 & 10.6 & 45.6 & & & & & & & \multirow[t]{2}{*}{0.065} \\
\hline & & Ischemic stroke & & & & 24.6 & 14.1 & 16.9 & 44.4 & & & & & & & \\
\hline \multirow{3}{*}{$\begin{array}{l}\quad \text { Red } \\
\text { meats/products, } \\
\text { Poultry/game }\end{array}$} & \multirow{2}{*}{$(-4)-(4)$} & $\begin{array}{l}\text { Non-Ischemic } \\
\text { stroke }\end{array}$ & & & & & 5.2 & 28.3 & 19.8 & 15.6 & 31.1 & & & & & \multirow{2}{*}{0.004} \\
\hline & & Ischemic stroke & & & & & 12.0 & 32.4 & 16.2 & 14.1 & 25.4 & & & & & \\
\hline & \multirow{2}{*}{$(-4)-(0)$} & $\begin{array}{l}\text { Non-Ischemic } \\
\text { stroke }\end{array}$ & & & & & 85.8 & 11.7 & 2.5 & & & & & & & \multirow{2}{*}{0.546} \\
\hline Fish/shrimps & & Ischemic stroke & & & & & 88.7 & 9.9 & 1.4 & & & & & & & \\
\hline \multirow[t]{2}{*}{ Eggs } & $(-4)-(4)$ & $\begin{array}{l}\text { Non-Ischemic } \\
\text { stroke }\end{array}$ & & & & & 49.4 & 34.1 & 13.3 & 1.4 & 1.8 & & & & & 0.896 \\
\hline & & Ischemic stroke & & & & & 49.3 & 36.6 & 12.0 & 0.7 & 1.4 & & & & & \\
\hline Cooking oils & $(0)-(6)$ & $\begin{array}{l}\text { Non-Ischemic } \\
\text { stroke }\end{array}$ & & & & & & & 36.2 & 22.4 & 12.4 & 29.0 & & & & $<0.001$ \\
\hline & & Ischemic stroke & & & & & & & 19.7 & 19.0 & 16.2 & 45.1 & & & & \\
\hline Alcoholic & $(0)-(6)$ & $\begin{array}{l}\text { Non-Ischemic } \\
\text { stroke }\end{array}$ & & & & & & & 93.8 & 3.2 & 1.3 & 1.7 & & & & 0.009 \\
\hline beverages & & Ischemic stroke & & & & & & & 91.5 & 1.4 & 4.2 & 2.8 & & & & \\
\hline Addible & $(0)-(6)$ & $\begin{array}{l}\text { Non-Ischemic } \\
\text { stroke }\end{array}$ & & & & & & & 98.9 & 0.7 & 0.1 & 0.3 & & & & 0.915 \\
\hline sugar & & Ischemic stroke & & & & & & & 99.3 & 0.7 & 0 & 0 & & & & \\
\hline Salt & $(0)-(6)$ & $\begin{array}{l}\text { Non-Ischemic } \\
\text { stroke }\end{array}$ & & & & & & & 39.5 & 41.0 & 5.1 & 14.4 & & & & 0.004 \\
\hline & & Ischemic stroke & & & & & & & 27.5 & 55.6 & 3.5 & 13.4 & & & & 0.007 \\
\hline Diet variety & $(-12)-(0)$ & $\begin{array}{l}\text { Non-Ischemic } \\
\text { stroke }\end{array}$ & 0 & 0.1 & 4.2 & 9.3 & 21.1 & 50.0 & 15.3 & & & & & & & 0.644 \\
\hline & & Ischemic stroke & 0 & 0 & 4.9 & 6.3 & 21.8 & 54.9 & 12.0 & & & & & & & \\
\hline
\end{tabular}

a Score range of total score is -60 to $44 ;{ }^{b} p$ value for chi-square test for the proportions of the scores for each food group.

The DBI-16 also revealed that 57.3\%, 35.1\%, and $2.3 \%$ of participants had a low, moderate, and high level of under intake (indicated by LBS), respectively; $43.8 \%, 16.8 \%$, and $0.9 \%$ of them had a low to high level of over intake (indicated by HBS), respectively; $50.6 \%$, $44.1 \%$, and $3.9 \%$ of them had a low to high-level problem of overall unbalance (indicated by DQD), respectively (Table 3). The ischemic stroke patients had a higher median HBS, higher prevalence of moderate level of over intake (HBS, 25.4\%) and higher overall unbalance (DQD, 48.6\%) as compared with those without ischemic stroke. Moreover, the distributions of dietary quality across two groups divided by area and ethnicity are shown in Figure 2. 
Table 3. Distribution of dietary quality and the percentages of participants with each category.

\begin{tabular}{|c|c|c|c|c|c|c|c|c|c|}
\hline \multirow[b]{2}{*}{$\begin{array}{c}\text { Diet } \\
\text { Quality }\end{array}$} & \multirow[b]{2}{*}{ Indicator } & \multirow[b]{2}{*}{$\begin{array}{l}\text { Score } \\
\text { Range }\end{array}$} & \multirow[b]{2}{*}{ Group } & \multirow[b]{2}{*}{ Mean \pm SD } & \multicolumn{5}{|c|}{ Distribution of Dietary Quality (\%) a } \\
\hline & & & & & $\begin{array}{l}\text { No } \\
\text { Prob- } \\
\text { lem }\end{array}$ & $\begin{array}{c}\text { Almost } \\
\text { No } \\
\text { Prob- } \\
\text { lem }\end{array}$ & $\begin{array}{c}\text { Low } \\
\text { Level } \\
\text { Prob- } \\
\text { lem }\end{array}$ & $\begin{array}{c}\text { Moderate } \\
\text { Level } \\
\text { Prob- } \\
\text { lem }\end{array}$ & $\begin{array}{l}\text { High } \\
\text { Level } \\
\text { Prob- } \\
\text { lem }\end{array}$ \\
\hline \multirow{2}{*}{$\begin{array}{l}\text { Under } \\
\text { intake }\end{array}$} & \multirow{2}{*}{ LBS } & \multirow{2}{*}{$0-60$} & $\begin{array}{l}\text { Non-Ischemic } \\
\text { stroke }\end{array}$ & $22.68 \pm 6.82$ & 0 & 5.3 & 57.2 & 35.2 & 2.3 \\
\hline & & & Ischemic stroke & $22.42 \pm 7.09$ & 0 & 5.6 & 62.0 & 29.6 & 2.8 \\
\hline \multirow[t]{2}{*}{ Over intake } & \multirow[t]{2}{*}{ HBS } & \multirow[t]{2}{*}{$0-40$} & $\begin{array}{l}\text { Non-Ischemic } \\
\text { stroke }\end{array}$ & $11.84 \pm 6.68$ & 2.7 & 36.0 & 43.8 & 16.6 & 0.9 \\
\hline & & & Ischemic stroke & $13.30 \pm 6.43$ & 2.1 & 28.9 & 42.9 & 25.4 & 0.7 \\
\hline \multirow{2}{*}{$\begin{array}{c}\text { Overall } \\
\text { unbalance }\end{array}$} & \multirow{2}{*}{ DQD } & \multirow{2}{*}{$0-84$} & $\begin{array}{l}\text { Non-Ischemic } \\
\text { stroke }\end{array}$ & $34.51 \pm 8.35$ & 0 & 1.4 & 50.7 & 44.0 & 3.9 \\
\hline & & & Ischemic stroke & $35.72 \pm 7.81$ & 0 & 0 & 47.2 & 48.6 & 4.2 \\
\hline
\end{tabular}

${ }^{a}$ Distribution of the lower bound score (LBS): No problem: 0; Almost no problem: 1-12; Low level: 13-24 Moderate level: 25-36; High level: 37-60. Distribution of the higher bound score (HBS): No problem: 0; Almost no problem: 1-9; Low level: 10-18; Moderate level: 19-27; High level: 28-44. Distribution of the diet quality distance (DQD): No problem: 0; Almost no problem: 1-17; Low level: 18-34; Moderate level: 35-50; High level: 51-84.

\subsection{Association Analyses of Ischemic Stroke with Dietary Quality Indicators and DBI-16 Components}

The results of Cox regression analyses were shown in Table 4. The hazard ratios (HRs) for ischemic stroke were progressively elevated with increasing HBS. Compared with participants in the lowest quartile (Q1) of HBS, those in the highest quartile (Q4) had a 3.15-fold (95\%CI: 1.50-6.63) increased risk for ischemic stroke, after adjustment for age, sex, area, ethnic group, education level, marriage status, smoking, diabetes, hypertension, dyslipidemia, and obesity status; (Model 2), and the association slightly increased after additional adjustment for medication use and nutraceutical intake at baseline (HR: 3.31, 95\%CI: 1.57-6.97, Model 3). A similar result was observed for the highest DQD quartile (Q4), with the corresponding multiple-adjusted HR of 2.19 (95\%CI: 1.24-3.86) based on Model 2 and 2.26 (95\%CI: 1.28-4.00) based on Model 3.

Table 4. Hazard ratios (HRs) and 95\% confidence intervals (95\%CIs) for ischemic stroke by diet quality indicators and DBI-16 components according to Cox regression models.

\begin{tabular}{|c|c|c|c|c|c|c|}
\hline \multirow{2}{*}{ Indicators } & \multirow{2}{*}{$\begin{array}{l}\text { No } \\
(n)\end{array}$} & \multirow{2}{*}{$\begin{array}{c}\text { Cases } \\
(n)\end{array}$} & \multirow{2}{*}{$\begin{array}{c}\text { Incident Density (Cases } \\
\text { per } 1000 \text { PYs) }\end{array}$} & \multicolumn{3}{|c|}{$\operatorname{HR}(95 \% C I)^{a}$} \\
\hline & & & & Model 1 & Model 2 & Model 2 \\
\hline \multicolumn{7}{|l|}{ LBS $^{b}$} \\
\hline Quartile 1 (Q1) & 1761 & 34 & 2.78 & 1.00 & 1.00 & 1.00 \\
\hline Quartile 2 (Q2) & 1907 & 41 & 3.06 & $1.04(0.66-1.64)$ & $1.13(0.68-1.89)$ & $1.13(0.68-1.89)$ \\
\hline Quartile 3 (Q3) & 2008 & 29 & 2.07 & $0.78(0.48-1.29)$ & $0.79(0.44-1.41)$ & $0.76(0.43-1.36)$ \\
\hline $\begin{array}{c}\text { Quartile } 4 \text { (Q4) } \\
\text { HBS c }^{\text {C }}\end{array}$ & 2165 & 38 & 2.46 & $0.92(0.57-1.46)$ & $0.86(0.46-1.59)$ & $0.84(0.45-1.56)$ \\
\hline Quartile 1 (Q1) & 1619 & 14 & 1.19 & 1.00 & 1.00 & 1.00 \\
\hline Quartile 2 (Q2) & 2042 & 37 & 2.57 & $2.24(1.21-4.15) *$ & $2.38(1.12-5.05)^{*}$ & $2.38(1.12-5.06)$ * \\
\hline Quartile 3 (Q3) & 2168 & 44 & 2.89 & $2.48(1.36-4.53)^{* *}$ & $2.38(1.14-5.00)^{*}$ & $2.39(1.14-5.01)$ * \\
\hline $\begin{array}{l}\text { Quartile } 4(\mathrm{Q} 4) \\
\text { DQD }^{\mathrm{d}}\end{array}$ & 2012 & 47 & 3.43 & $3.12(1.72-5.68)^{* * *}$ & $3.15(1.50-6.63)^{* * *}$ & $3.31(1.57-6.97)^{* *}$ \\
\hline Quartile 1 (Q1) & 1853 & 26 & 1.98 & 1.00 & 1.00 & 1.00 \\
\hline Quartile 2 (Q2) & 1797 & 32 & 2.50 & $1.28(0.76-2.15)$ & $1.34(0.75-2.37)$ & $1.33(0.75-2.36)$ \\
\hline Quartile 3 (Q3) & 2166 & 36 & 2.36 & $1.29(0.78-2.13)$ & $1.11(0.62-2.01)$ & $1.13(0.63-2.04)$ \\
\hline Quartile 4 (Q4) & 2025 & 48 & 3.44 & $1.99(1.23-3.23)^{* *}$ & $2.19(1.24-3.86)^{* *}$ & $2.26(1.28-4.00)^{* *}$ \\
\hline
\end{tabular}


Table 4. Cont.

\begin{tabular}{|c|c|c|c|c|c|c|}
\hline \multirow{2}{*}{ Indicators } & \multirow{2}{*}{$\begin{array}{l}\text { No } \\
(n)\end{array}$} & \multirow{2}{*}{$\begin{array}{c}\text { Cases } \\
(n)\end{array}$} & \multirow{2}{*}{$\begin{array}{c}\text { Incident Density (Cases } \\
\text { per } 1000 \text { PYs) }\end{array}$} & \multicolumn{3}{|c|}{$\operatorname{HR}(95 \% C I)^{a}$} \\
\hline & & & & Model 1 & Model 2 & Model 2 \\
\hline \multicolumn{7}{|l|}{ Cereals } \\
\hline Score 0 & 1252 & 23 & 2.61 & 1.00 & 1.00 & 1.00 \\
\hline $\begin{array}{c}\text { Score } \\
(-12)-(-7)\end{array}$ & 220 & 3 & 1.90 & $0.76(0.23-2.51)$ & $1.06(0.31-3.62)$ & $1.03(0.30-3.53)$ \\
\hline Score $(-6)-(-1)$ & 680 & 11 & 2.26 & $0.85(0.41-1.74)$ & $0.69(0.29-1.65)$ & $0.65(0.27-1.55)$ \\
\hline Score (1)-(6) & 2192 & 31 & 2.01 & $0.74(0.43-1.26)$ & $0.55(0.29-1.04)$ & $0.54(0.28-1.02)$ \\
\hline Score (7)-(12) & 3497 & 74 & 3.02 & $1.08(0.67-1.72)$ & $0.95(0.56-1.61)$ & $0.94(0.55-1.59)$ \\
\hline \multicolumn{7}{|l|}{ Vegetables } \\
\hline Score 0 & 2982 & 56 & 2.67 & 1.00 & 1.00 & 1.00 \\
\hline \multicolumn{7}{|l|}{ Fruits } \\
\hline Score 0 & 354 & 4 & 1.62 & 1.00 & 1.00 & 1.00 \\
\hline $\begin{array}{c}\text { Score }(-6)-(-1) \\
\text { Dairy }\end{array}$ & 7487 & 138 & 2.62 & $1.75(0.65-4.73)$ & $1.88(0.59-6.04)$ & $1.95(0.61-6.28)$ \\
\hline Score 0 & 25 & 1 & 5.84 & 1.00 & 1.00 & 1.00 \\
\hline \multicolumn{7}{|l|}{ Soybeans } \\
\hline Score 0 & 3575 & 63 & 2.52 & 1.00 & 1.00 & 1.00 \\
\hline \multicolumn{7}{|l|}{ Meats } \\
\hline Score 0 & 1544 & 23 & 2.12 & 1.00 & 1.00 & 1.00 \\
\hline Score $(-4)-(-1)$ & 2645 & 63 & 3.4 & $1.66(1.03-2.68)$ * & $1.13(0.67-1.90)$ & 1.08 (0.64-1.82) \\
\hline Score (1)-(4) & 3652 & 56 & 2.17 & $0.95(0.59-1.55)$ & $0.64(0.37-1.12)$ & $0.63(0.36-1.09)$ \\
\hline \multicolumn{7}{|l|}{ Fish/shrimps } \\
\hline Score 0 & 194 & 2 & 1.47 & 1.00 & 1.00 & 1.00 \\
\hline $\begin{array}{c}\text { Score }(-4)-(-1) \\
\text { Eggs }\end{array}$ & 7647 & 140 & 2.60 & $1.76(0.44-7.10)$ & $2.02(0.28-14.50)$ & $1.98(0.28-14.20)$ \\
\hline Score 0 & 1042 & 17 & 2.37 & 1.00 & 1.00 & 1.00 \\
\hline Score $(-4)-(-1)$ & 6547 & 122 & 2.64 & $1.01(0.61-1.68)$ & $0.78(0.45-1.35)$ & $0.79(0.46-1.37)$ \\
\hline Score (1)-(4) & 252 & 3 & 1.69 & $0.65(0.19-2.21)$ & $0.52(0.12-2.25)$ & $0.50(0.12-2.20)$ \\
\hline \multicolumn{7}{|l|}{ Cooking oils } \\
\hline Score 0 & 2814 & 28 & 1.39 & 1.00 & 1.00 & 1.00 \\
\hline \multicolumn{7}{|l|}{$\begin{array}{l}\text { Alcoholic } \\
\text { beverages }\end{array}$} \\
\hline Score 0 & 7353 & 130 & 2.51 & 1.00 & 1.00 & 1.00 \\
\hline Score (1)-(6) & 488 & 12 & 3.64 & $1.62(0.90-2.93)$ & $1.30(0.60-2.80)$ & $1.35(0.62-2.93)$ \\
\hline \multicolumn{7}{|l|}{ Addible sugar } \\
\hline Score 0 & 7757 & 141 & 2.59 & 1.00 & 1.00 & 1.00 \\
\hline $\begin{array}{l}\text { Score (1)-(6) } \\
\text { Salt }\end{array}$ & 84 & 1 & 1.74 & $0.81(0.11-5.80)$ & $0.84(0.12-6.00)$ & $0.81(0.11-5.81)$ \\
\hline Score 0 & 3079 & 39 & 1.76 & 1.00 & 1.00 & 1.00 \\
\hline Score (1)-(6) & 4762 & 103 & 3.13 & $2.04(1.41-2.96)^{* * *}$ & $1.98(1.29-3.02)^{* *}$ & $2.03(1.33-3.10)^{* *}$ \\
\hline \multicolumn{7}{|l|}{ Dietary variety } \\
\hline Score 0 & 1195 & 17 & 1.97 & 1.00 & 1.00 & 1.00 \\
\hline $\begin{array}{c}\text { Score } \\
(-12)-(-7)\end{array}$ & 337 & 7 & 2.85 & $1.58(0.65-3.85)$ & $5.24(1.66-16.50)^{* *}$ & $5.40(1.70-17.20)^{* *}$ \\
\hline Score $(-6)-(-1)$ & 6309 & 118 & 2.68 & $1.65(0.99-2.76)$ & $1.66(0.94-2.95)$ & $1.69(0.95-3.01)$ \\
\hline
\end{tabular}

${ }^{a}$ Model 1: Adjusted for age only; Model 2: Model $1+$ additionally adjusted for sex, area, ethnic group, education level, marriage status, economic level, smoking, diabetes, hypertension, dyslipidemia, and obesity status; Model 3: Model $2+$ additionally adjusted for medication use and nutraceutical intake. ${ }^{\mathrm{b}}$ Quartile levels for the lower bound score (LBS): Q1, score 0-18; Q2, score 19-22; Q3, score 23-27; Q4, score 28-52. ' Quartile levels for the higher bound score (HBS): Q1, score 0-6; Q2, score 7-12; Q3, score 13-17; Q4, score 18-33. ${ }^{\mathrm{d}}$ Quartile levels for the diet quality distance (DQD): Q1, score 9-29; Q2, score 30-34; Q3, score 35-40; Q4, score 41-68. * $p<0.05$; ** $p<0.01$; $* * * p<0.001$ 


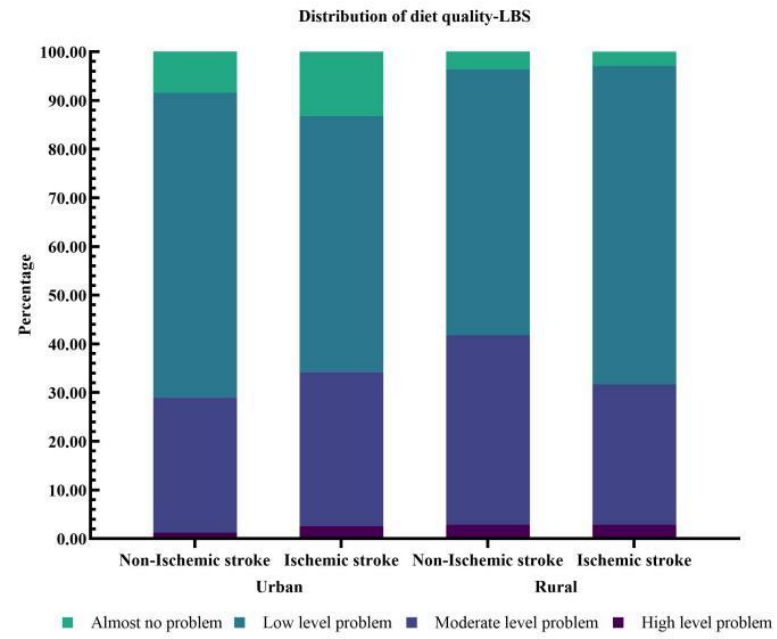

(A)

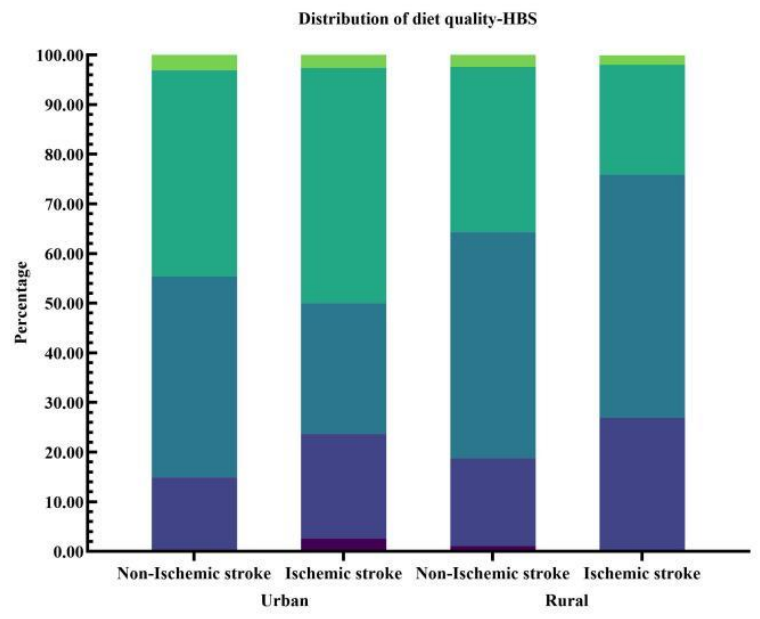

(C)

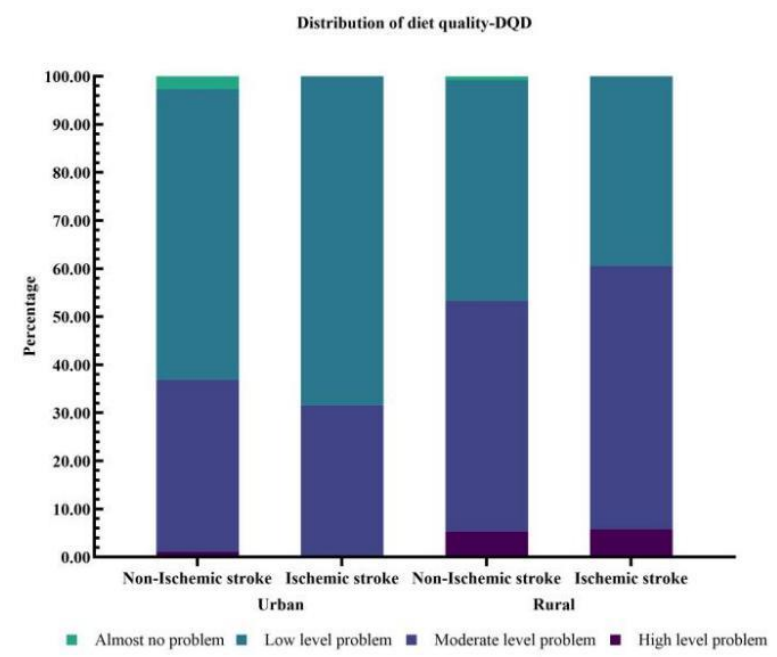

(E)

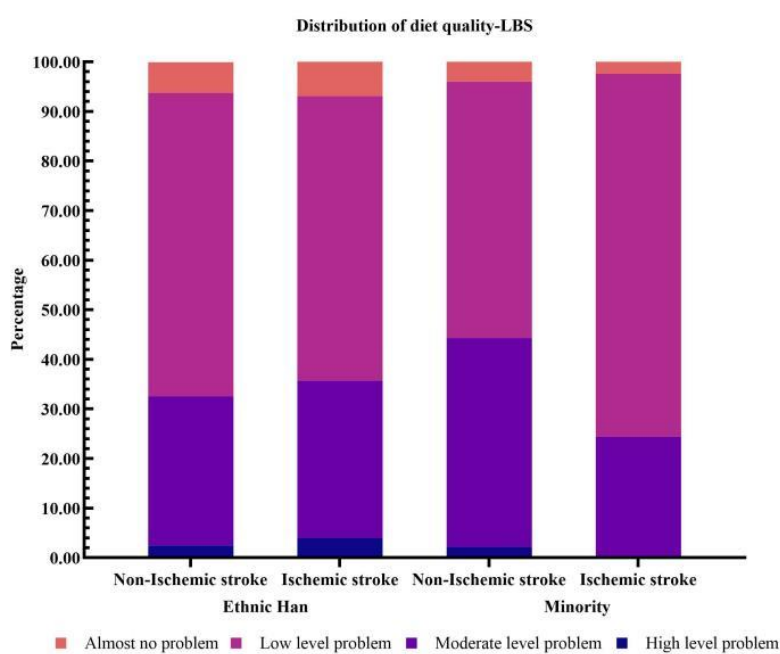

(B)

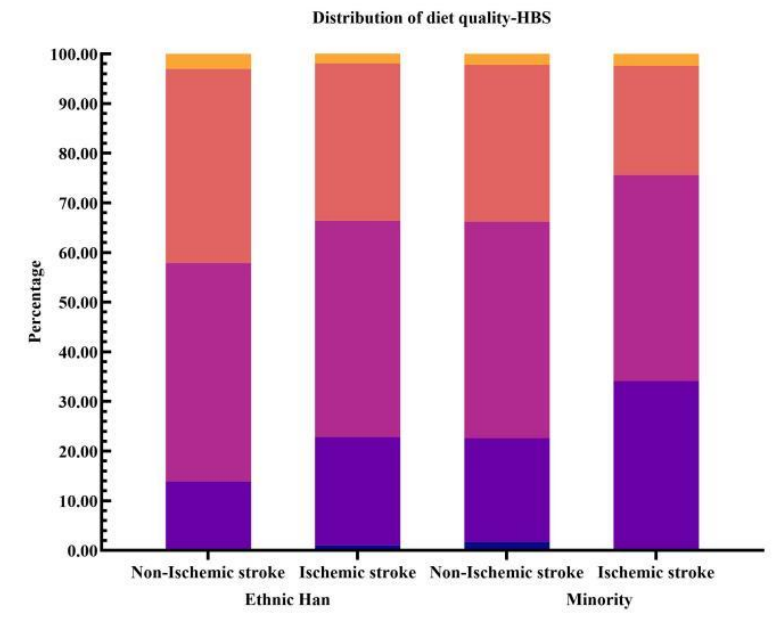

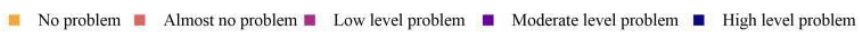

(D)

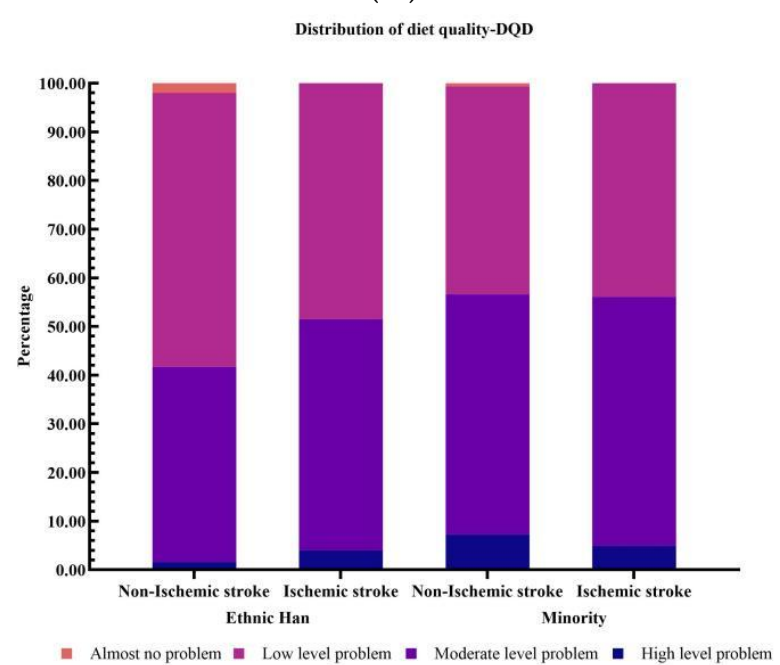

(F)

Figure 2. Distribution of the diet quality stratified by area (urban/rural) and ethnic group (ethnic Han/minority): (A,B) for the lower bound score (LBS); (C,D) for the higher bound score (HBS); $(\mathrm{E}, \mathrm{F})$ for the diet quality distance (DQD). 
Among the thirteen components of DBI-16, both cooking oils and salt showed significant associations with ischemic stroke. Compared with the appropriate intake level (score $=0)$, excessive intake level (score 1-6) in cooking oils or salt added a $200 \%$ risk (HR: 3.00, 95\%CI: 1.77-5.07, Model 3) and 103\% risk (HR: 2.03, 95\%CI: 1.33-3.10, Model 3) for ischemic stroke, respectively. Moreover, lower dietary variety (score -12 to -7 ) also promoted incident ischemic stroke (HR:5.40, 95\%CI: 1.70-17.20, Model 3).

3.4. Stratified Analyses of the Association of Ischemic Stroke with Dietary Quality Indicators across Different Status of Comorbidities, Medication Use, and Nutraceutical Intake

After eliminating the role of comorbidities, medications, and nutraceuticals, HBS was still related to ischemic stroke among participants without diabetes or obesity, or those free of nutraceuticals or medications (Figure 3). In addition, the effect strengths of the associations between HBS with ischemic stroke were more evident in those with hypertension history (HR: 7.10, 95\%CI: 2.71-19.9) and using medications (HR: 6.30, 95\%CI: 1.44-18.6) than in total participants, comparing the highest quartile (Q4) of HBS to the lowest quartile (Q1) based on Model 3. However, HBS seemed to play less of a role in ischemic stroke among those with diabetes or intaking nutraceuticals. A similar pattern was also observed for DQD, with the increased HRs of 4.87 (95\%CI: 1.66-14.20, Q4 vs. Q1, Model 3) and 6.00 (95\%CI: 1.39-17.70, Q4 vs. Q1, Model 3) among those with hypertension history and using medications, respectively.
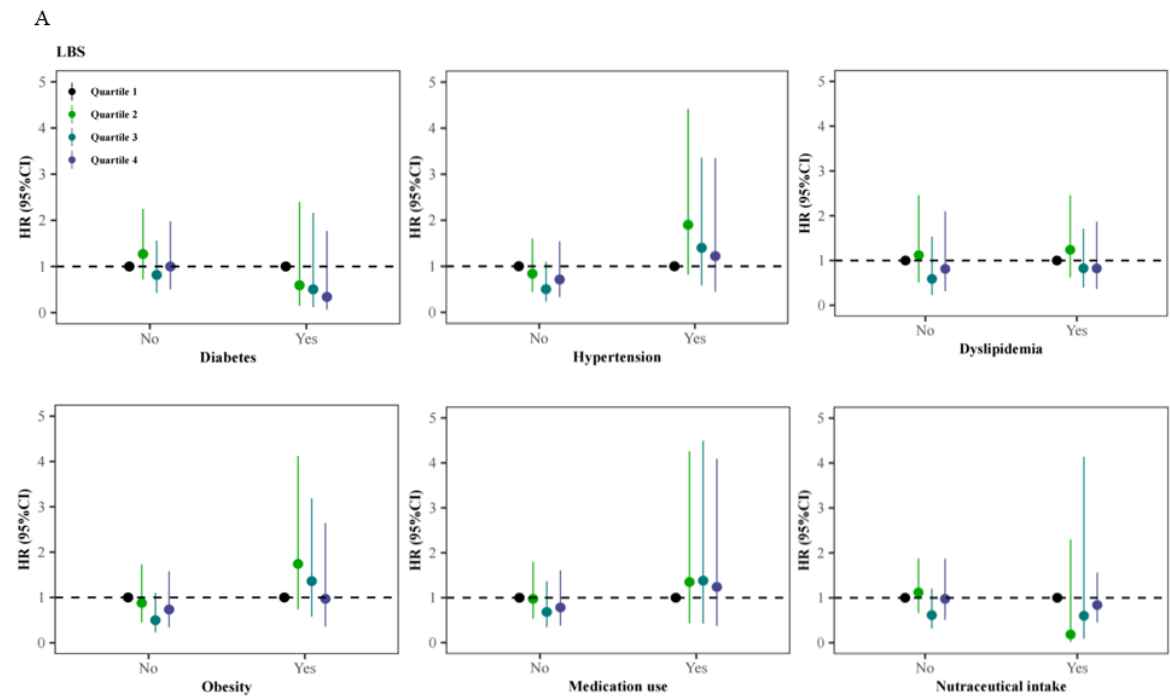

B
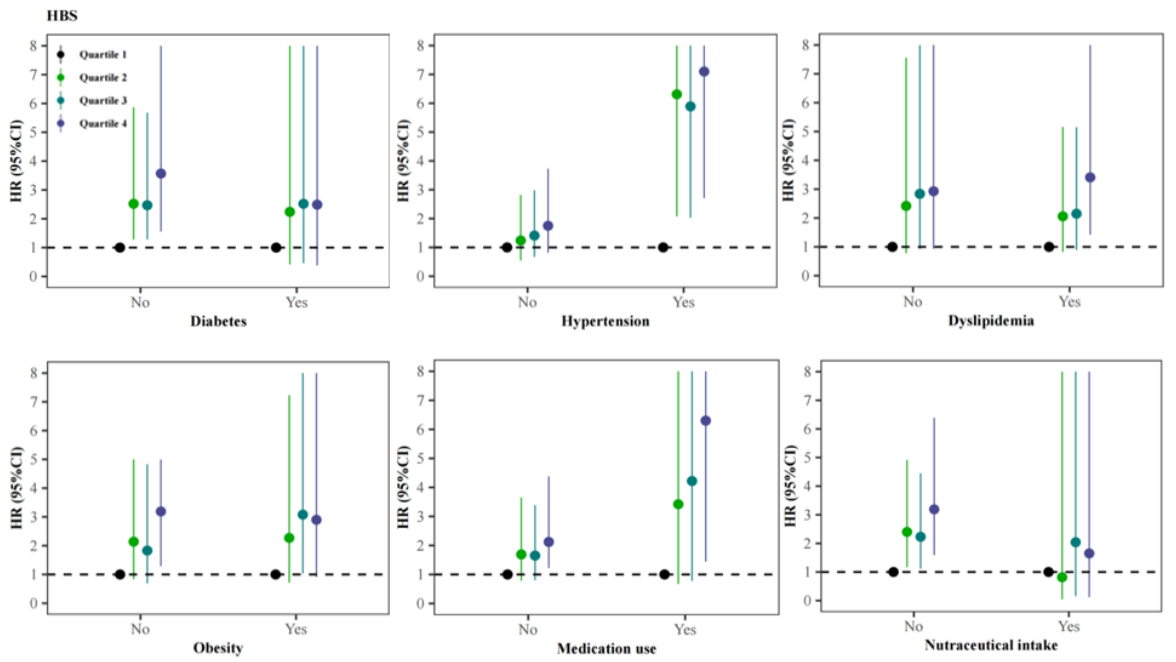

Figure 3. Cont. 

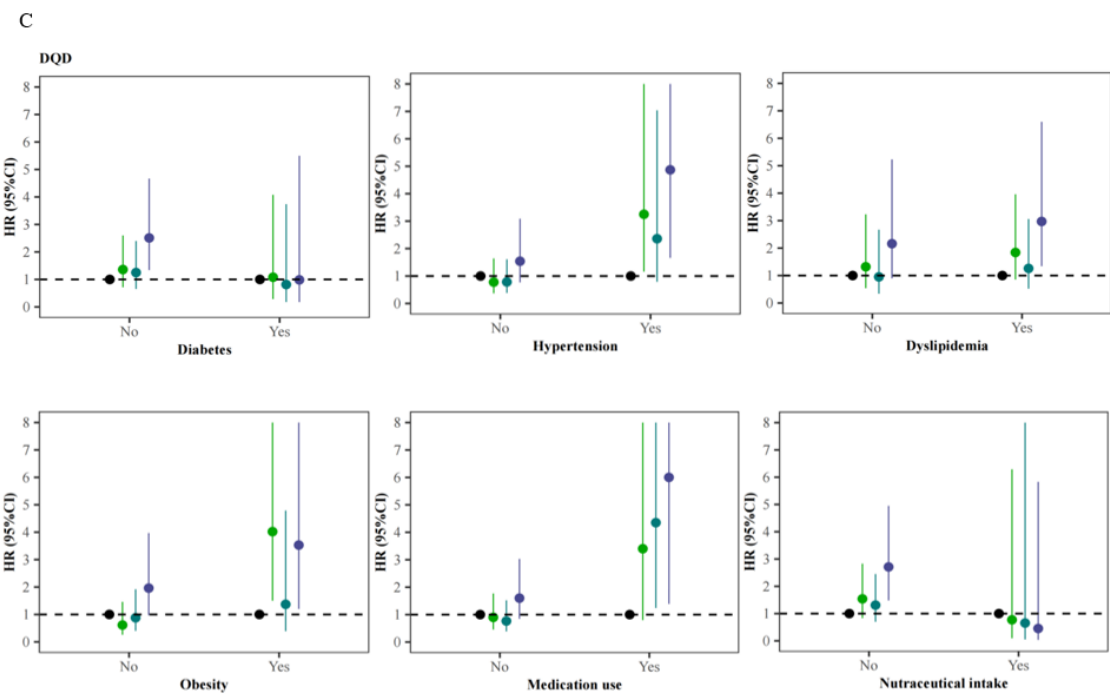

Figure 3. Adjusted hazard ratios (HRs) and 95\% confidence intervals (95\%CIs) for ischemic stroke associated with baseline dietary quality after stratified by the status of diabetes, hypertension, dyslipidemia, obesity, medication use and nutraceutical intake: (A) for the lower bound score (LBS); (B) for the higher bound score (HBS); (C) for the diet quality distance (DQD).

\subsection{Stratified Analyses of the Associations of Ischemic Stroke with Dietary Quality Indicators across Baseline Demographic Factors}

The multi-adjusted HRs for ischemic stroke by dietary quality indicators varied according to different demographic factors (Figure 4). The positive associations between HBS and ischemic stroke were seen only among participants with a baseline age of 60 years or more (HR: 4.70, 95\%CI: 2.89-16.00, Q4 vs. Q1), female (HR: 2.94, 95\%CI: 1.17-7.34, Q4 vs. Q1), rural residents (HR: 3.50, 95\%CI: 1.57-7.81, Q4 vs. Q1) and the Ethnic Han (HR: 3.15, 95\%CI: 1.36-7.32, Q4 vs. Q1), although there was no significant effect modification $\left(p_{\text {interaction }}>0.05\right)$ by age, sex, area, and ethnic group. When predicted by DQD, the risks for ischemic stroke elevated in females (HR: 2.68, 95\%CI: 1.24-5.80, Q4 vs. Q1), rural residents (HR: 2.49, 95\%CI: 1.26-4.90, Q4 vs. Q1), and the Ethnic Han (HR: 2.80, 95\%CI: 1.36-5.77, Q4 vs. Q1). There was additionally a negative association between LBS and ischemic stroke in those aged more than 60 years (HR: 0.12, 95\%CI: 0.03-0.55, Q3 vs. Q1). 
A

- Quartile2 vs Quartile1 • Quartile3 vs Quartilel • Quartile4 vs Quartile

LBS

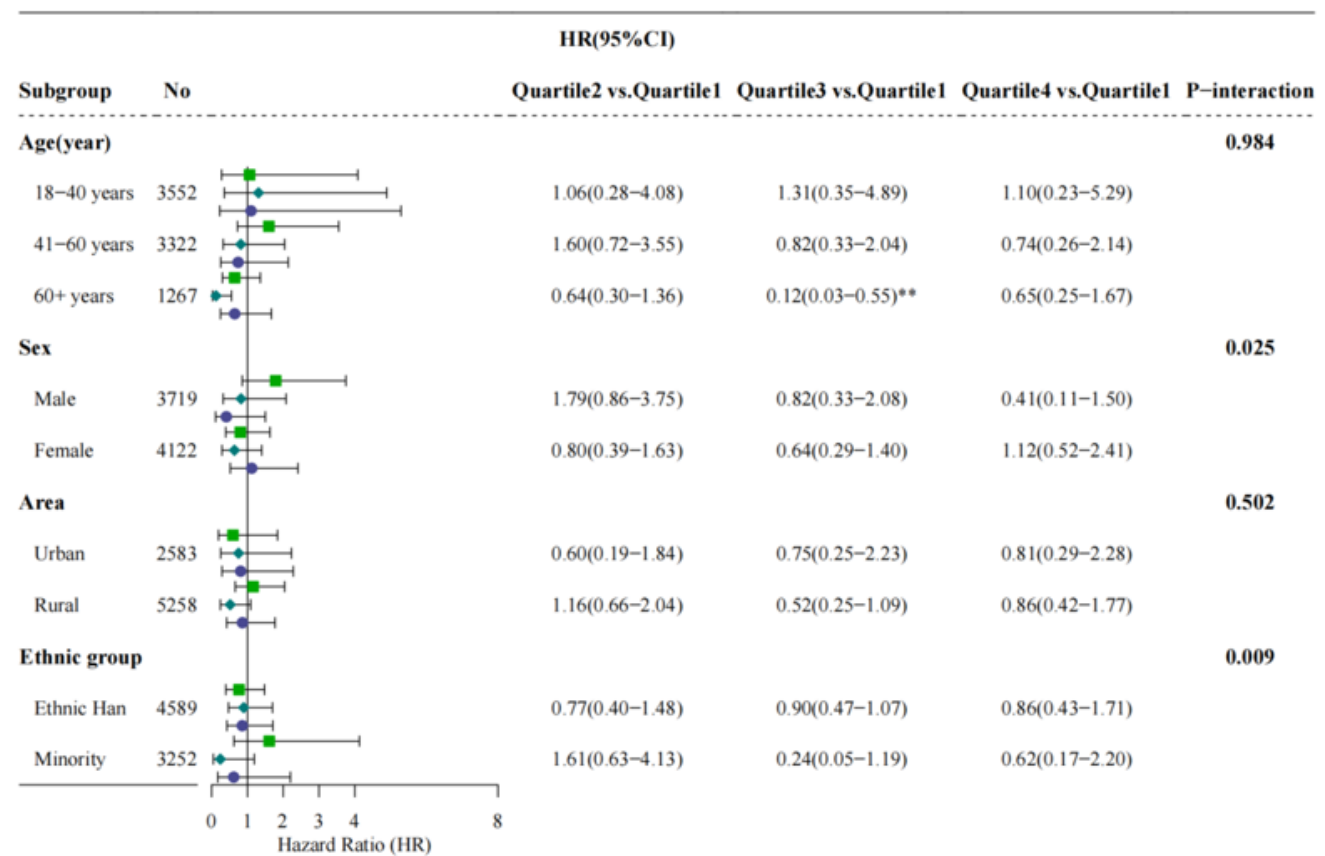

B

- Quartile2 vs Quartilel • Quartile3 vs.Quartilel • Quartile4 vs Quartilel

HBS

HR $(95 \% \mathrm{CI})$

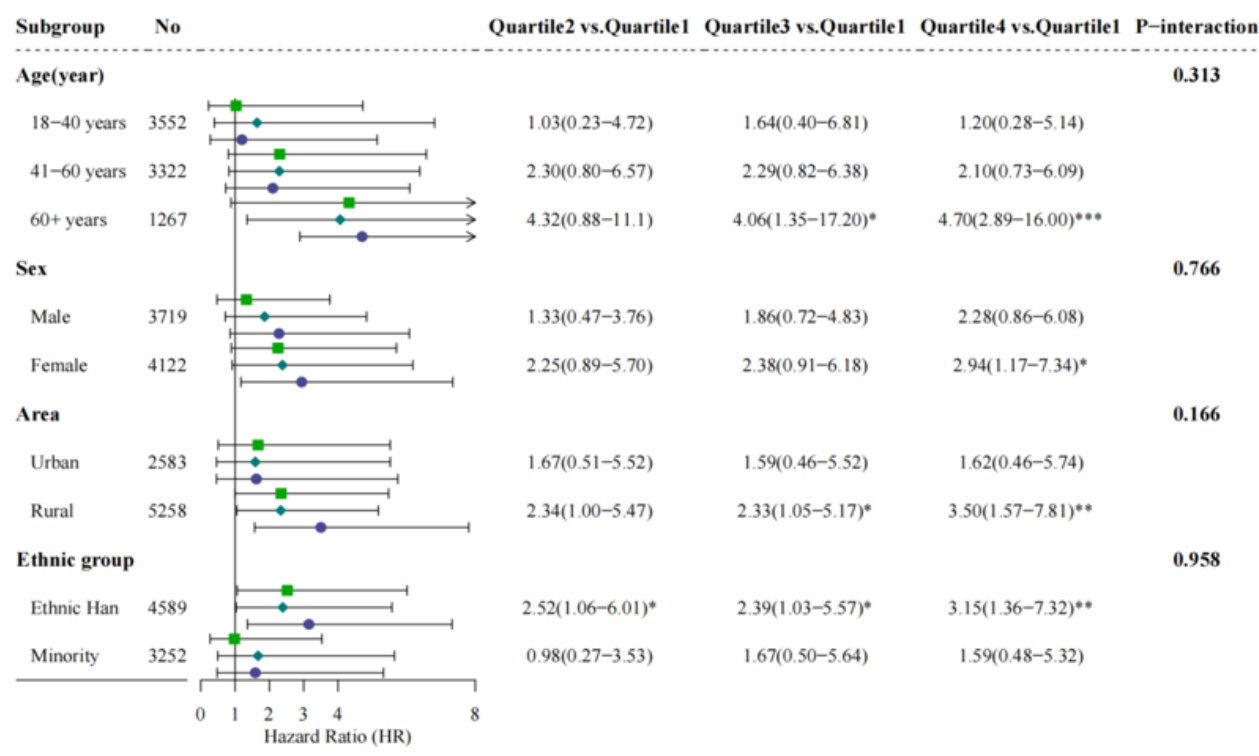

Figure 4. Cont. 


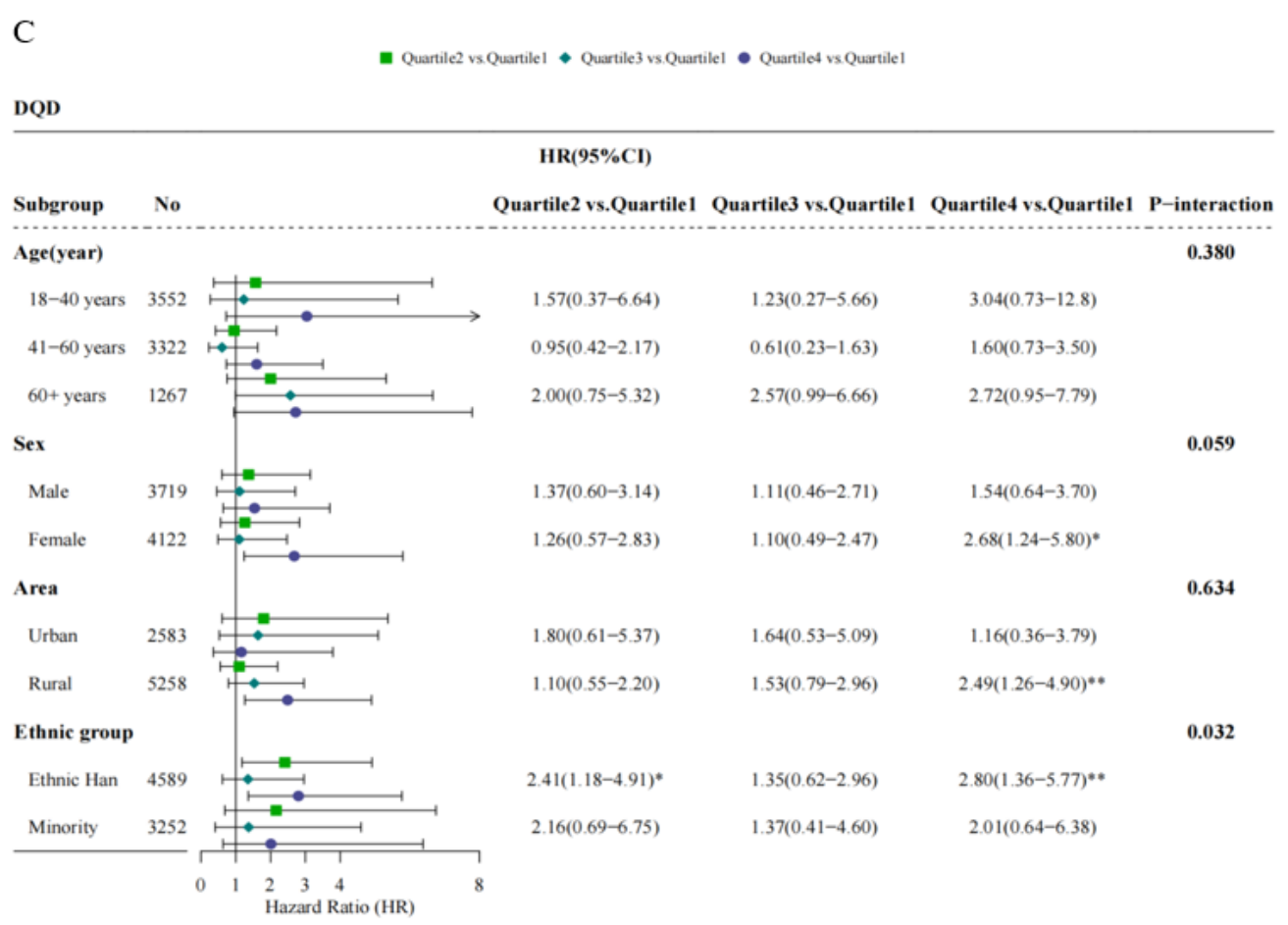

Figure 4. Adjusted hazard ratios (HRs) and 95\% confidence intervals (95\%CIs) for ischemic stroke associated with baseline dietary quality after stratified by age, sex, area, and ethnic group: (A) for the lower bound score (LBS); (B) for the higher bound score (HBS); (C) for the diet quality distance (DQD); ${ }^{*} p<0.05 ;{ }^{* *} p<0.01 ;{ }^{* * *} p<0.001$.

\section{Discussions}

In this prospective cohort study conducted in Guizhou Province, Southwest China, we observed that the participants were exposed to dietary unbalance to different extents at baseline, mainly including the inadequate intakes of dairy, fish, fruits, eggs, vegetables, and soybeans, and the excessive intakes of cereals, cooking oils, salt, and meats. Our analyses further suggested that unfavorable dietary quality, including overall excessive consumption, high oils and salt diet, and low food diversity, may be a risk for ischemic stroke.

Several studies conducted in American, Swedish, and Chinese populations have estimated the effects of dietary quality on ischemic stroke. A recent study based on 73,890 women in Nurses' Health Study (NHS, 1984-2016), 92,352 women in NHSII (19912017), and 43,266 men in Health Professionals Follow-Up Study (1986-2012) in America revealed that the healthful plant-based dietary quality assessed by the Plant-based Diet Index (PDI) seemed to reduce the risk of ischemic stroke [22]. Similarly, a prospective cohort study of 26,547 Swedish aged 46 to 73 years found that less risk of incident ischemic stroke was related to the higher dietary quality, which was based on adherence to the Swedish nutrition recommendations [23]. By contrast, a large-scale prospective cohort study of 512,725 Chinese aged 30 to 79 years reported that less healthy dietary habits, which was defined as non-daily eating of vegetables, fruits, and eggs combined with eating daily or less than weekly, contributed to incident ischemic stroke [24].

China has experienced an ongoing transition of dietary patterns over the past decades, which mainly featured declines in the intakes of coarse and refined grains and vegetables, as well as increases in the intakes of animal-derived foods, with pork being most popular. Intakes of eggs, fish, and dairy have been consistently below recommended levels [25]. In addition, as the cooking methods markedly shifted from steaming and boiling to stirfried and deep-fried, the daily consumption of cooking oils has been gradually increased from $18.2 \mathrm{~g}$ to $42.1 \mathrm{~g}$ from 1982 to 2012 [26]. People preferred to add a certain amount of salt to keep food from spoiling, such as pickles and salted fish, especially in rural or remote areas without a good condition for food storage. Data from China National Nutrition 
Surveys (CHNS) also revealed that more than $55.9 \%$ and $71.8 \%$ of Chinese have consumed excessive cooking oil and salt, which were far above the recommended levels and strongly linked to increased risk of chronic diseases [27]. These cooking and eating behaviors are partly driven both by the great accessibility of cooking oils and salt and by their low price.

Compared to a previous study from Shanghai in East China and a cross-sectional study based on the CHNS, participants in this current study from Guizhou Province in Southeast China have a higher percentage of excessive intake of cooking oil and salt (score $>0$ ) [28,29]. We also observed that the probabilities of occurring ischemic stroke for participants consuming the excessive level of cooking oil and salt were about appropriate two to three times as high as those consuming the moderate level. Cooking oil has been the main source of fat intake these decades. As expected, a diet with elevated fat or salt was associated with a significantly increased risk of hypertension and stroke, as reported previously [30,31]. A rat experiment found that a long-term administration of canola oil, sesame oil, or trans-fat led to marked dyslipidemia, fat accumulation, neuroinflammation, vascular lesion, and endothelial injury for rats, and thereby remarkably contributed to ischemic and hemorrhagic strokes [32]. Results from a gerbil animal model study indicated that a high-fat diet accelerates and exacerbates microgliosis and neuronal damage [33]. Although extra virgin olive oil (EVOO) or other oils rich in monounsaturated fat have been reported to bring some benefits to the cardiocerebral vascular system, but their consumption by the Chinese was relatively low [26]. In numerous epidemiology and clinical studies, excessive dietary sodium salt intake was linked to hypertension, which has been considered as the main risk factor for stroke [34]. A large prospective cohort study observed a significant linear association between calibrated urinary sodium excretion and stroke in patients with chronic kidney disease [35].

Given the potential effects of comorbidities, some medications for metabolic diseases, and some nutraceuticals, which may promote or decelerate the progress of cardiovascular diseases [36,37], we performed the same analyses in those with different status' of these conditions. The risk effects of excessive food intake (evaluated by HBS) and unbalanced food intake were more evident in those with hypertension history and taking medications for metabolic diseases, indicating that people with a high risk of stroke should pay more attention to the balance of food types and daily intake, reasonable diet is also one of the major measures to prevent hypertension [16]. However, we failed to observe any associations of ischemic stroke with diet quality among those with diabetes or intaking nutraceuticals, perhaps due to a smaller sample size in those subgroups.

The current study provides primary evidence that the risk of ischemic stroke among the residents from Guizhou Province in Southwest China may be partly due to the potential effects of dietary quality. The chief strengths lie in the use of a large population-based cohort, a prospective study design with more than six years of follow-up, a dietary assessment index suitable for Chinese people. The scores of DBI-16 are based on different levels of energy consumption for specific individuals recommended by the most recent Dietary Guidelines for Chinese residents, so the potential confounding effects of total energy intake may be appropriately controlled [38]. There also exist certain limitations. Firstly, dietary habits and socioeconomic characteristics were collected based on individual self-report, which might lead to recall bias. The food frequency questionnaire (FFQ) has been considered a convenient and widely-used dietary assessment tool, but this method is subject to less accuracy of quantification of food portions than the method of weighing, which might make some measurement errors [39]. Secondly, we just applied the proportion of condiment intakes at home to estimate the total daily intakes, inevitably ignoring the condiment intakes from eating outside. Thirdly, our study population was from Southeast China and the dietary assessment index was applicable to the Chinese, so the findings from the current study should be generalized with caution to other populations. In conclusion, considering a great disease burden caused by ischemic stroke in China, our study suggests that it is essential to adjust dietary habits and conduct dietary interventions, especially 
controlling risk factors in preventive mode better than therapeutic mode, because of a fairly short time to death or irreversible injuries after the onset of stroke.

\section{Conclusions}

Our results suggest that unfavorable dietary quality, including overall excessive consumption, excessive intake of cooking oils and salt, or under adequate dietary diversity, may increase the risk for ischemic stroke.

Supplementary Materials: The following are available online at: https:/ / www.mdpi.com/article / 10.3390/nu14030694/s1, Table S1: DBI-16 components and standard for scoring.

Author Contributions: Conceptualization, T.L., N.W. and C.F.; Data curation, X.S. and Y.C.; Formal analysis, Y.W. (Yingying Wang) and N.W.; Funding acquisition, T.L.; Investigation, Y.W. (Yiying Wang) and J.Z.; Methodology, Y.W. (Yingying Wang) and N.W.; Project administration, T.L. and C.F.; Supervision, C.F.; Writing—original draft, Y.W. (Yingying Wang); Writing-review \& editing, X.S. and N.W. All authors have read and agreed to the published version of the manuscript.

Funding: This work was supported by Guizhou Province Science and Technology Support Program (Qiankehe [2018]2819).

Institutional Review Board Statement: The study was conducted according to the guidelines of the Declaration of Helsinki and approved by the Institutional Review Board (or Ethics Committee) of the Guizhou Center for Disease Control and Prevention (no. S2017-02).

Informed Consent Statement: Informed consent was obtained from all subjects that were involved in the study.

Data Availability Statement: The datasets for this manuscript will be made available upon request pending, further inquiries can be directed to the corresponding author Liu Tao, liutaombs@163.com and Na Wang, na.wang@fudan.edu.cn.

Conflicts of Interest: The authors declare that they have no known competing financial interest or personal relationship that could have appeared to influence the work reported in this paper.

\section{References}

1. Tsao, C.W.; Aday, A.W.; Almarzooq, Z.I.; Alonso, A.; Beaton, A.Z.; Bittencourt, M.S.; Boehme, A.K.; Buxton, A.E.; Carson, A.P.; Commodore-Mensah, Y.; et al. Heart Disease and Stroke Statistics-2021 Update: A Report From the American Heart Association. Circulation 2021, 143, e254-e743.

2. Wang, Y.-J.; Li, Z.-X.; Gu, H.-Q.; Zhai, Y.; Jiang, Y.; Zhao, X.-Q.; Wang, Y.-L.; Yang, X.; Wang, C.-J.; Meng, X.; et al. China Stroke Statistics 2019: A Report From the National Center for Healthcare Quality Management in Neurological Diseases, China National Clinical Research Center for Neurological Diseases, the Chinese Stroke Association, National Center for Chronic and Non-communicable Disease Control and Prevention, Chinese Center for Disease Control and Prevention and Institute for Global Neuroscience and Stroke Collaborations. Stroke Vasc. Neurol. 2020, 5, 211-239. [PubMed]

3. Li, Z.; Jiang, Y.; Li, H.; Xian, Y.; Wang, Y. China's response to the rising stroke burden. BMJ 2019, 364, 1879. [CrossRef]

4. Wang, W.; Jiang, B.; Sun, H.; Ru, X.; Sun, D.; Wang, L.; Wang, L.; Jiang, Y.; Li, Y.; Wang, Y.; et al. Prevalence, Incidence, and Mortality of Stroke in China: Results from a Nationwide Population-Based Survey of 480687 Adults. Circulation 2017, 135, 759-771. [CrossRef] [PubMed]

5. National Center for Cardiovascular Diseases. Annual Report on Cardiovascular Health and Diseases in China 2019; China Science Publishing House: Beijing, China, 2020. (In Chinese)

6. Lv, J.; Yu, C.; Guo, Y.; Bian, Z.; Yang, L.; Chen, Y.; Tang, X.; Zhang, W.; Qian, Y.; Huang, Y.; et al. Adherence to Healthy Lifestyle and Cardiovascular Diseases in the Chinese Population. J. Am. Coll. Cardiol. 2017, 69, 1116-1125. [CrossRef]

7. Chao, A.M.; Quigley, K.M.; Wadden, T.A. Dietary interventions for obesity: Clinical and mechanistic findings. J. Clin. Investig. 2021, 131, e140065. [CrossRef]

8. Ojo, O. Dietary Intake and Type 2 Diabetes. Nutrients 2019, 11, 2177. [CrossRef]

9. Badimon, L.; Chagas, P.; Chiva-Blanch, G. Diet and Cardiovascular Disease: Effects of Foods and Nutrients in Classical and Emerging Cardiovascular Risk Factors. Curr. Med. Chem. 2019, 26, 3639-3651. [CrossRef] [PubMed]

10. Krebs-Smith, S.M.; Pannucci, T.E.; Subar, A.F.; Sharon, R.D.; Kirkpatrick, I.; Lerman, J.L.; Tooze, J.; Wilson, M.M.; Reedy, J. Update of the Healthy Eating Index: HEI-2015. J. Acad. Nutr. Diet. 2018, 118, 1591-1602. [CrossRef]

11. Cruz, R.D.; Park, S.-Y.; Shvetsov, Y.B.; Boushey, C.J.; Monroe, K.R.; Le Marchand, L.; Maskarinec, G. Diet Quality and Breast Cancer Incidence in the Multiethnic Cohort. Eur. J. Clin. Nutr. 2020, 74, 1743-1747. [CrossRef] 
12. Turati, F.; Carioli, G.; Bravi, F.; Ferraroni, M.; Serraino, D.; Montella, M.; Giacosa, A.; Toffolutti, F.; Negri, E.; Levi, F.; et al. Mediterranean Diet and Breast Cancer Risk. Nutrients 2018, 10, 326. [CrossRef]

13. He, Y.N.; Fang, Y.H.; Xia, J. Update of the Chinese Diet Balance Index: DBI-16. Acta Nutr. Sin. 2018, 40, 526-530.

14. Chinese Nutrition Society. Dietary Guidelines for Chinese Residents (2016); People's Medical Publishing House: Beijing, China, 2016. (In Chinese)

15. Tan, S.; Lu, H.; Song, R.; Wu, J.; Xue, M.; Qian, Y.; Wang, W.; Wang, X. Dietary quality is associated with reduced risk of diabetes among adults in Northern China: A cross-sectional study. Br. J. Nutr. 2021, 126, 923-932. [CrossRef]

16. Wang, X.; Liu, A.; Du, M.; Wu, J.; Wang, W.; Qian, Y.; Zheng, H.; Liu, D.; Nan, X.; Jia, L.; et al. Diet quality is associated with reduced risk of hypertension among Inner Mongolia adults in northern China. Public Health Nutr. 2020, 23, 1543-1554. [CrossRef] [PubMed]

17. Gao, X.; Tian, Z.; Zhao, D.; Li, K.; Zhao, Y.; Xu, L. Associations between Adherence to Four A Priori Dietary Indexes and Cardiometabolic Risk Factors among Hyperlipidemic Patients. Nutrients 2021, 13, 2179. [CrossRef] [PubMed]

18. Ma, R.C.W. Epidemiology of diabetes and diabetic complications in China. Diabetologia 2018, 61, 1249-1260. [CrossRef] [PubMed]

19. He, Y.N.; Zhai, F.Y.; Yang, X.G.; Ge, K.Y. The Chinese Diet Balance Index Revised. Acta Nutr. Sin. 2009, 31, 532-536.

20. Writing group of Chinese Guideline for Cardiometabolic Diseases Prevention; Editorial board of Chinese Journal of Cardiometabolic Diseases. Chinese Guideline for Cardiometabolic Diseases Prevention (2017). Chin. J. Cardiometabolic Dis. 2018, 46, $10-25$.

21. National Health and Family Planning Commission of the People's Republic of China. Health Standard of the People's Republic of China; No. WS/T 428-2013; Criteria of Weight for Adults 2013; Standards Press of China: Beijing, China, 2013. (In Chinese)

22. Baden, M.Y.; Shan, Z.; Wang, F.; Li, Y.; Manson, J.E.; Rimm, E.B.; Willett, W.C.; Hu, F.B.; Rexrode, K.M. Quality of Plant-Based Diet and Risk of Total, Ischemic, and Hemorrhagic Stroke. Neurology 2021, 96, e1940-e1953. [CrossRef]

23. Johansson, A.; Drake, I.; Engström, G.; Acosta, S. Modifiable and Non-Modifiable Risk Factors for Atherothrombotic Ischemic Stroke among Subjects in the Malmö Diet and Cancer Study. Nutrients 2021, 13, 1952. [CrossRef]

24. Han, Y.; Hu, Y.; Yu, C.; Guo, Y.; Pei, P.; Yang, L.; Chen, Y.; Du, H.; Sun, D.; Pang, Y.; et al. Lifestyle, cardiometabolic disease, and multimorbidity in a prospective Chinese study. Eur. Heart J. 2021, 42, 3374-3384. [CrossRef]

25. Zhai, F.; Du, S.F.; Wang, Z.; Zhang, J.G.; Du, W.; Popkin, B.M. Dynamics of the Chinese diet and the role of urbanicity, $1991-2011$. Obes. Rev. 2014, 15 (Suppl. 1), 16-26. [CrossRef] [PubMed]

26. Huang, L.; Wang, Z.; Wang, H.; Zhao, L.; Jiang, H.; Zhang, B.; Ding, G. Nutrition transition and related health challenges over decades in China. Eur J. Clin. Nutr. 2021, 75, 247-252. [CrossRef] [PubMed]

27. Jiang, H.; Zhang, J.; Su, C.; Zhang, J.; Zhang, B.; Wang, H. Cooking oil and salt consumption among chinese adults aged 18-59 years in 2015. Acta Nutr. Sin. 2018, 40, 27-31.

28. Zang, J.; Yu, H.; Zhu, Z.; Lu, Y.; Liu, C.; Yao, C.; Bai, P.; Guo, C.; Jia, X.; Zou, S.; et al. Does the Dietary Pattern of Shanghai Residents Change across Seasons and Area of Residence: Assessing Dietary Quality Using the Chinese Diet Balance Index (DBI). Nutrients 2017, 9, 251. [CrossRef] [PubMed]

29. He, D.; Qiao, Y.; Xiong, S.; Liu, S.; Ke, C.; Shen, Y. Association between Dietary Quality and Prediabetes based on the Diet Balance Index. Sci. Rep. 2020, 10, 3190. [CrossRef]

30. Muto, M.; Ezaki, O. High Dietary Saturated Fat is Associated with a Low Risk of Intracerebral Hemorrhage and Ischemic Stroke in Japanese but not in Non-Japanese: A Review and Meta-Analysis of Prospective Cohort Studies. J. Atheroscler. Thromb. 2018, 25, 375-392. [CrossRef]

31. Arsang-Jang, S.; Mansourian, M.; Mohammadifard, N.; Khosravi, A.; Oveis-Gharan, S.; Nouri, F.; Sarrafzadegan, N. Temporal trend analysis of stroke and salt intake: A 15-year population-based study. Nutr. Neurosci. 2021, 24, 384-394. [CrossRef]

32. Guo, H.; Ban, Y.-H.; Cha, Y.; Kim, T.-S.; Lee, S.-P.; An, E.S.; Choi, J.; Seo, D.W.; Yon, J.-M.; Choi, E.-K.; et al. Comparative effects of plant oils and trans-fat on blood lipid profiles and ischemic stroke in rats. J. Biomed. Res. 2017, 31, 122-129.

33. Seo, W.J.; Ahn, J.H.; Lee, T.-K.; Kim, B.; Lee, J.-C.; Park, J.H.; Yoo, Y.H.; Shin, M.C.; Cho, J.H.; Won, M.-H.; et al. High fat diet accelerates and exacerbates microgliosis and neuronal damage/death in the somatosensory cortex after transient forebrain ischemia in gerbils. Lab. Anim. Res. 2020, 36, 28. [CrossRef]

34. Frisoli, T.M.; Schmieder, R.E.; Grodzicki, T.; Messerli, F.H. Salt and hypertension: Is salt dietary reduction worth the effort? Am. J. Med. 2012, 125, 433-439. [CrossRef]

35. Mills, K.T.; Chen, J.; Yang, W.; Appel, L.J.; Kusek, J.W.; Alper, A.; Delafontaine, P.; Keane, M.G.; Mohler, E.; Ojo, A.; et al. Sodium Excretion and the Risk of Cardiovascular Disease in Patients With Chronic Kidney Disease. JAMA 2016, 315, $2200-2210$. [CrossRef] [PubMed]

36. Li, X.; Wu, C.; Lu, J.; Chen, B.; Li, Y.; Yang, Y.; Hu, S.; Li, J. Cardiovascular risk factors in China: A nationwide population-based cohort study. Lancet Public Health 2020, 5, e672-e681. [CrossRef]

37. Scicchitano, P.; Cameli, M.; Maiello, M.; Modesti, P.A.; Muiesan, M.L.; Novo, S.; Palmiero, P.; Saba, P.S.; Pedrinelli, R.; Ciccone, M.M. Nutraceuticals and dyslipidaemia: Beyond the common therapeutics. J. Funct. Foods 2014, 6, 11-32. [CrossRef] 
38. Zhang, Q.; Qin, G.; Liu, Z.; Li, Z.; Li, J.; Varma, D.S.; Wan, Q.; Zhao, J.; Min, X.; Han, X.; et al. Dietary Balance Index-07 and the Risk of Anemia in Middle Aged and Elderly People in Southwest China: A Cross Sectional Study. Nutrients 2018, 10, 162. [CrossRef]

39. McKenzie, B.L.; Coyle, D.H.; Santos, J.A.; Burrows, T.; Rosewarne, E.; Peters, S.A.E. Investigating sex differences in the accuracy of dietary assessment methods to measure energy intake in adults: A systematic review and meta-analysis. Am. J. Clin. Nutr. 2021, 113, 1241-1255. [CrossRef] [PubMed] 